\title{
Improving HPV Vaccine Delivery: A National Study of Parents and their Adolescent Sons
}

\author{
Paul L. Reiter, PhD ${ }^{1,2}$, Annie-Laurie McRee, DrPH ${ }^{3}$, Jessica K. Pepper, MPH ${ }^{4}$, Kim \\ Chantala, $\mathbf{M S}^{4}$, and Noel T. Brewer, $\mathbf{P h D}^{4,5}$ \\ ${ }^{1}$ Division of Cancer Prevention and Control, College of Medicine, The Ohio State University, \\ Columbus, $\mathrm{OH}$ \\ ${ }^{2}$ Comprehensive Cancer Center, The Ohio State University, Columbus, $\mathrm{OH}$ \\ ${ }^{3}$ Department of Pediatrics, University of Minnesota, Minneapolis, MN \\ ${ }^{4}$ UNC Gillings School of Global Public Health, Chapel Hill, NC \\ ${ }^{5}$ Lineberger Comprehensive Cancer Center, Chapel Hill, NC
}

\begin{abstract}
Purpose-We examined parents' and adolescents' preferences regarding potential strategies to increase HPV vaccination rates, including offering the vaccine in alternative settings, concomitant administration of vaccines, and optimizing the structure of vaccination medical visits.
\end{abstract}

Methods-A national sample of US parents of adolescent males ages 11-17 years ( $n=506)$ and their sons ( $n=391$ ) completed online surveys in August and September 2010. We used analysis of variance for mixed designs to examine preferences for vaccination settings.

Results-Parents and sons were most comfortable with sons getting HPV vaccine in a doctor's office. Parents of sons who had not visited their regular healthcare providers in the last year were more comfortable with sons getting HPV vaccine at a public clinic $(p<0.001)$ or school $(p<0.05)$ compared to parents whose sons had recent visits. Results from the son survey showed a similar pattern. Parents and sons reported moderate levels of acceptability of concomitant administration. They most preferred to have the three HPV vaccine shots administered during brief nurse visits.

Conclusion-Offering HPV vaccine in alternative settings and administering it with other recommended adolescent vaccines may increase uptake among adolescent males. Parents and sons may prefer for HPV vaccination to occur during brief nurse visits.

(C) 2012 Society for Adolescent Medicine. Published by Elsevier Inc. All rights reserved.

Correspondence and requests for reprints should be sent to: Paul L. Reiter, PhD, Division of Cancer Prevention and Control, College of Medicine, The Ohio State University, Suite 525, 1590 North High Street, Columbus, Ohio 43201, 614-366-4265 phone,

614-293-5611 fax, Paul.Reiter@osumc.edu.

Implications and Contribution

Offering HPV vaccine in alternative settings and administering it with other recommended adolescent vaccines may increase uptake among adolescent males. To our knowledge, this study provides the first national data on this range of HPV vaccine delivery preferences for adolescent males.

\section{Conflict of Interest}

A research grant to NTB and PLR from Merck Sharp \& Dohme Corp. funded the study. Merck Sharp \& Dohme Corp. played no role in the study design, planning, implementation, analysis, or reporting of the findings. NTB has also received grants and/or honoraria from GlaxoSmithKline and Merck Sharp \& Dohme Corp. PLR has not received honoraria or consulting fees from these companies.

Publisher's Disclaimer: This is a PDF file of an unedited manuscript that has been accepted for publication. As a service to our customers we are providing this early version of the manuscript. The manuscript will undergo copyediting, typesetting, and review of the resulting proof before it is published in its final citable form. Please note that during the production process errors may be discovered which could affect the content, and all legal disclaimers that apply to the journal pertain. 


\section{Keywords}

HPV; vaccine; school; pharmacy; adolescents

\section{Introduction}

Since 2006, guidelines have recommend routine provision of quadrivalent HPV vaccine for 11-12 year-old females with catch-up vaccination for females through age 26 (1).

Guidelines also now recommend routine vaccination for males ages 11-12 with catch-up vaccination to age 21 (2). Despite recommendations, only 49\% of 13-17 year-old females and about $2 \%$ of adolescent males have received any doses of HPV vaccine, though estimates for males precede the recommendation for routine male vaccination $(3,4)$. One potentially effective strategy to increase these rates is to offer HPV vaccine in alternative settings, such as schools and pharmacies. School-located HPV vaccination programs have been highly effective in Australia and the United Kingdom (5-7) and have just started to develop in the United States (US) (8). Pharmacies represent a setting beyond the traditional medical home that may also help improve vaccination rates (9). As of 2008, 49 states allowed pharmacists to vaccinate adults and 27 allowed some form of adolescent vaccination (10). Most pharmacy representatives believe retail pharmacies will expand adolescent vaccination services in the future (11).

Other aspects of vaccine delivery, such as concomitant administration of vaccines and optimizing the structure of vaccination medical visits, may also help increase HPV vaccination. HPV vaccine can be administered at the same visit as other appropriate vaccines (1), and our past research showed that adolescent females who had received meningococcal vaccine were more likely to have also received HPV vaccine (12). Administering HPV vaccine during adolescents' medical visits when healthcare providers deliver other services (e.g., routine screenings or physical exams) is a standard practice that provides another possible avenue for increasing vaccination.

Such strategies could be particularly important to increasing HPV vaccination among adolescent males. Adolescent males, especially older adolescents, may receive fewer outpatient healthcare visits overall and fewer preventive care visits compared to adolescent females (13). Physicians may also be less likely to recommend HPV vaccination to male adolescents compared to females, though most providers indicate they would recommend vaccination for males (14).

Limited research has examined parents' and adolescents' vaccine delivery preferences. Parents have expressed modest to high acceptance of school-located vaccination for adolescents (including HPV vaccination), with less acceptance of pharmacy-located vaccination (15-18). Adolescents have expressed modest acceptance for both school- and pharmacy-located vaccination (18). Many parents and adolescents would be willing for the adolescents to receive multiple vaccines during the same medical visit (18). For vaccines requiring three medical visits, such as HPV vaccine, most parents have indicated they prefer to have at least one of the visits involve other adolescent healthcare services (19). We are not aware of any studies, however, examining this range of vaccine delivery preferences among a national sample of parents and adolescents. In the current study, we examined HPV vaccine delivery preferences of US parents and their adolescent sons. 


\section{Materials and Methods}

\section{Study Design}

The HPV Immunization in Sons (HIS) study examined HPV vaccine attitudes and beliefs of parents and their adolescent sons ages 11-17 years (4). We recruited parents from a national panel of US households maintained by an online survey company (Knowledge Networks). The survey company used a dual frame approach (list-assisted, random-digit dialing supplemented by address-based sampling) to sample households into their panel (20). Panel members in non-Internet households receive a laptop and free internet access for completing multiple online surveys each month. In households with existing internet access, panel members receive points for completing surveys that can later be redeemed for small cash payments. The survey company randomly sampled panel members with sons ages 11-17 years to participate. We asked participating parents to allow their sons to participate in the study as well. The Institutional Review Board at the University of North Carolina approved the study.

A total of 1195 parents received email invitations to participate in our study, of whom 752 responded. Of these parents, $73 \%$ ( $n=547)$ were eligible and completed the parent survey. We asked parents with multiple sons ages 11-17 to answer questions about their son who had the most recent birthday and to permit this son to complete the son survey. Over half of responding parents $(56 \%, n=421)$ had sons who completed the son survey. Parents and sons completed surveys in August and September 2010 after providing consent (parents) or assent (sons) to participate. Parents with existing computer and internet access received about 1,000 points ( $\$ 1.00$ equivalent) for completing our survey and sons received points equivalent to $\$ 5$.

We do not report data on parents whose sons had already received one or more doses of HPV vaccine $(n=12)$ or vaccinated sons who completed surveys $(n=9)$. We also do not report data on parents whose sons were home-schooled $(n=29)$ or their sons $(n=21)$, as they did not receive items about school-located HPV vaccination. Thus, our analytic sample included 506 parents and 391 sons.

A majority of parents were younger than 45 years of age $(61 \%)$ and non-Hispanic white $(67 \%)$ (Table 1). About half of parents were female (54\%), had at least some college education (56\%), and reported a household income of at least $\$ 60,000(50 \%)$. Most sons who completed surveys were non-Hispanic white $(61 \%)$, with fairly equal distribution across the three age groups (30\% ages $11-12,38 \%$ ages $13-15$, and $31 \%$ ages $16-17$ ). About $79 \%$ of sons had seen their regular healthcare provider in the last year. Parents and sons reported moderate levels of overall HPV vaccine acceptability, results we reported previously (4).

\section{Measures}

The parent and son surveys are available online at http://www.unc.edu/ ntbrewer/hpv.htm. We developed survey items concerning HPV vaccination in alternative settings based on our previous research among parents with adolescent daughters (15).

Parent and son surveys included corresponding items regarding possible settings for HPV vaccination. All items started with the phrase, "If you and (son's name) decided to get him the HPV vaccine..." for the parent survey and, "If you and your parents decided to get you the HPV vaccine..." for the son survey. Surveys asked participants how comfortable they were with the sons getting HPV vaccine in four settings: (a) a local pharmacy or drug store if it offered the vaccine; (b) the son's school if it offered the vaccine in a nurse's office; (c): a public or community clinic (a clinic where people get medical care at low cost that is not a 
private doctor's office); and (d) a doctor's office. A preamble to the items clarified that comfortable did not mean whether the shots themselves would be painful. All items had a 5point response scale ranging from "very uncomfortable" to "very comfortable" (coded 1-5 with higher values indicating greater comfort). Following the pharmacy and school items, surveys asked what concerns parents and sons would have about sons getting HPV vaccine in these two settings, as well as what the potential benefits would be. We provided parents and sons with lists of concerns and potential benefits, from which they could select multiple answers.

Surveys assessed the types of healthcare visits at which respondents would prefer sons receive the three-dose HPV vaccine series (if they decided to vaccinate at a doctor's office): (a) brief nurse visits for all three shots; (b) longer doctor visits for all three shots that each include other preventive care services (such as a physical exam or counseling); or (c) a longer doctor visit that includes the first shot and other preventive care services, and brief nurse visits for the last two shots (19).

Surveys assessed acceptability of concomitant administration by asking participants how willing they would be for sons to get other vaccines during the same visit as HPV vaccine (supposing they had decided to vaccinate and a doctor recommended concomitant administration). Concomitant administration items had a 5-point response scale ranging from "definitely not willing" to "definitely willing" (coded 1-5 with higher values indicating greater willingness). We also collected information on parent and son demographic characteristics (Table 1).

\section{Data Analysis}

We analyzed parent and son data separately, with analyses conducted in SPSS version 17.0 (SPSS Inc., Chicago IL) and SAS Version 9.2 (Cary, NC). Because we thought preferences may differ depending on sons' recent healthcare utilization, we conducted a $4 \times 2$ analysis of variance for mixed designs to explore the influence of vaccination setting (within subjects, 4 levels) and whether sons had seen their regular healthcare providers in the last year (between subjects, 2 levels). We used McNemar's test to determine if concerns and perceived benefits of school- and pharmacy-located HPV vaccination differed. Statistical tests were two-tailed with a critical alpha of 0.05 .

\section{Results \\ HPV Vaccination Settings}

Parents were more comfortable with their sons getting HPV vaccine at a doctor's office than in other settings (Figure 1, panel A). The ratings for doctor's office (mean=4.58, standard deviation $[\mathrm{SD}]=0.79)$ were higher than for a public clinic (mean=3.08, $\mathrm{SD}=1.27)$, school (mean=2.90, $\mathrm{SD}=1.36$ ), or pharmacy (mean=2.88, $\mathrm{SD}=1.27$ ) (all $p<0.001)$. Parents whose sons had not seen their regular healthcare providers in the last year provided more favorable ratings of some alternative vaccination settings (interaction, $p=0.001$ ). Compared to parents whose sons had seen their regular healthcare providers in the last year, parents of sons without recent visits were more comfortable with them getting HPV vaccine at a public clinic $(p<0.001)$ or school $(p<0.05)$. Results also suggested these parents may have been somewhat more comfortable with pharmacy-located HPV vaccination $(p=0.08)$ and less comfortable with their sons getting HPV vaccine at a doctor's office $(p=0.14)$, though neither difference reached statistical significance.

Results from the son survey showed the same pattern (Figure 1, panel B). Sons were more comfortable getting HPV vaccine at a doctor's office (mean=4.24, $\mathrm{SD}=0.90$ ) compared to a public clinic $($ mean $=2.93, \mathrm{SD}=1.16)$, school $($ mean=2.62, $\mathrm{SD}=1.33)$, or pharmacy 
(mean=2.57, $\mathrm{SD}=1.22)$ (all $p<0.001)$. We again found a statistically significant interaction between vaccination setting and whether sons had seen their regular healthcare provider in the last year ( $p=0.003$ ). Compared to sons with recent provider visits, sons who had not seen their regular healthcare providers in the last year were more comfortable getting HPV vaccine at a public clinic $(p<0.05)$, school $(p<0.05)$, or pharmacy $(p<0.05)$. These sons appeared to be somewhat less comfortable getting HPV vaccine at a doctor's office $(p=0.12)$, though this difference again did not reach statistical significance.

\section{Concerns and Perceived Benefits}

Parents expressed similar concerns regarding school- and pharmacy-located HPV vaccination for their sons (Table 2). The most common concern was wanting their sons' doctors to keep track of their shots and other health history (school=65\%, pharmacy $=68 \%$ ). Other common concerns included training of staff and wanting to be present during vaccination. Sons were concerned about embarrassment of getting vaccinated at these locations, wanting their doctors to keep track of their shots and other health history, training of staff, and wanting a parent present during vaccination. Both parents and sons expressed more concern about pharmacies than schools with respect to staff training, while both groups were more concerned that schools should not provide vaccines and other people potentially finding out if vaccination occurred at schools (all $p<0.05$ ).

Parents perceived the main benefit of school- and pharmacy-located HPV vaccination for their sons would be its convenience (school=36\%, pharmacy=37\%) (Table 3). Sons indicated similar perceived benefits as parents regarding school- and pharmacy-located HPV vaccination. Both parents and sons more commonly noted these benefits for vaccinating at schools compared to pharmacies (all $p<0.05$ ): the chance for a lot of people to get vaccinated, sons could go with their friends to get vaccinated, and they would know the people giving the vaccine. Over $40 \%$ of parents and sons perceived no benefits of school- or pharmacy-located HPV vaccination.

\section{Vaccine Administration}

Parents (40\%) and sons (67\%) most preferred brief nurse visits for all three HPV vaccine shots, if sons were going to receive them at a doctor's office. Fewer preferred a longer doctor visit for the first shot (that included other preventive services) followed by brief nurse visits for the last two shots (parents $=39 \%$, sons $=16 \%$ ) or longer doctor visits for all three shots that each included other preventive care services (parents $=19 \%$, sons $=14 \%$ ). A few parents (2\%) and sons (3\%) did not indicate their preference.

Most parents (53\%) were definitely or probably willing to let their sons receive other vaccines during the same visit that they received HPV vaccine, if recommended by a doctor. About $28 \%$ of parents were not sure, and $19 \%$ were definitely or probably not willing. About $37 \%$ of sons were definitely or probably willing to receive other vaccines during the same visit as HPV vaccine if recommended by a doctor, $34 \%$ were not sure, and $29 \%$ were definitely or probably not willing.

\section{Discussion}

HPV vaccination coverage remains suboptimal among adolescent females and is nearly nonexistent among adolescent males in the US $(3,4)$. Potential strategies to increase HPV vaccination include offering the vaccine in alternative settings, concomitant administration of vaccines, and optimizing the structure of vaccination medical visits. These strategies may be especially beneficial to adolescent males, since older adolescent males receive fewer 
healthcare visits than females, and healthcare providers may be less likely to recommend HPV vaccination to adolescent males $(13,14)$.

Among a national sample of parents and their adolescent sons, we found participants were most comfortable with sons getting HPV vaccine in a doctor's office, though they were moderately comfortable with HPV vaccination in alternative settings. It is not surprising that comfort levels were highest for HPV vaccination at a doctor's office since this is the medical home for most adolescents (17), and over $90 \%$ of adolescent females who have received HPV vaccine did so at a family doctor/general practitioner or pediatrician's office (21). It is encouraging that a meaningful number of parents and sons were also comfortable with sons getting HPV vaccine in alternative settings, particularly in cases where sons had not had recent healthcare provider visits. These findings correspond with past studies examining views on school- and pharmacy-located vaccination for adolescents $(17,18)$. Our results suggest that offering HPV vaccine in alternative settings may help increase vaccination among adolescents who do not have regular healthcare visits, an important finding because low-income adolescents and those without health insurance are less likely to have preventive care visits (22).

Parents and sons expressed some concerns about HPV vaccination in alternative settings. Mainly, they were concerned about wanting sons' doctors to keep track of their vaccinations, the training of staff administering the vaccines, and wanting parents present during vaccination. These findings are similar to our previous research examining mothers' concerns about school-located HPV vaccination for their adolescent daughters (15). Many parents and sons, however, perceived potential benefits of these same alternative settings, including convenience and the ability to vaccinate a lot of people. If vaccination programs in alternative settings are to become more prevalent and successful, they will need to address concerns and stress the potential benefits.

In this study, parents and sons most preferred brief nurse visits for all three HPV vaccine doses. These findings are in contrast to past research in the US that found only $8 \%$ of parents preferred three brief nurse visits and most preferred to have at least one visit involve other adolescent preventive care services (19). This previous study, however, involved a hypothetical three-dose adolescent vaccine, so our results provide useful insight into parents' and sons' preferences for actual HPV vaccination. Future research is needed to determine if parents and adolescents are also willing for HPV vaccination to occur during other types of adolescent care visits (e.g., acute care visits).

Participants in our study reported moderate levels of acceptability of concomitant administration. This is encouraging because multiple other vaccines have recently been recommended for adolescents (e.g., tetanus booster, meningococcal, and seasonal influenza vaccines) (23-25), some of which are required for school entry (26). HPV vaccine can be administered at the same visit as other adolescent vaccines and doing so will likely increase the number of adolescents receiving vaccines on schedule (1). It is worth noting that a past study found higher levels of acceptability of concomitant administration of adolescent vaccines (18), though participants were recruited during medical visits at primary care practices and may have been more accepting of health-related behaviors in general.

Study strengths included using a national sample with data from both parents and sons and examining multiple strategies for increasing HPV vaccination. Although the online panel is comparable to the US population on many sociodemographic characteristics (27), most participants were non-Hispanic white and of fairly high socioeconomic status. Data collection occurred prior to the recommendation for routine HPV vaccination of males (2), and we do not know the generalizability of findings to adolescent females. Participants may 
have perceived items addressing HPV vaccination in alternative settings as somewhat hypothetical since school- and pharmacy-located vaccination is not widespread in the US. The survey also did not address other potentially important issues of HPV vaccination in alternative settings, such as the process of obtaining consent for vaccination.

Despite these limitations, we believe our findings have potentially important implications. HPV vaccine uptake may be increased by offering the vaccine in alternative settings and administering it with other recommended adolescent vaccines. Parents and sons may also prefer for HPV vaccination to occur during brief nurse visits. Together, these findings offer valuable insight into parents' and sons' preferences regarding HPV vaccination.

\section{Acknowledgments}

Funding

Supported in part by a research grant from the Investigator-Initiated Studies Program of Merck Sharp \& Dohme Corp. The opinions expressed in this paper are those of the authors and do not necessarily represent those of Merck Sharp \& Dohme Corp. Additional support provided by the American Cancer Society (MSRG-06-259-01-CPPB) and the Cancer Control Education Program at UNC Lineberger Comprehensive Cancer Center (R25 CA57726).

\section{Abbreviations}

CDC Centers for Disease Control and Prevention

US United States

HIS study HPV Immunization in Sons (HIS) study

SD standard deviation

\section{References}

1. Markowitz LE, Dunne EF, Saraiya M, et al. Quadrivalent human papillomavirus vaccine: recommendations of the Advisory Committee on Immunization Practices (ACIP). MMWR Recomm Rep. 2007; 56(RR-2):1-24. [PubMed: 17380109]

2. Centers for Disease Control and Prevention (CDC). CDC online newsroom: ACIP recommends all 11-12 year-old males get vaccinated against HPV. 2011. Available at: http://www.cdc.gov/media/releases/2011/t1025_hpv_12yroldvaccine.html

3. Centers for Disease Control and Prevention (CDC). National and state vaccination coverage among adolescents aged 13 through 17 years --- United States, 2010. MMWR Morb Mortal Wkly Rep. 2011; 60:1117-23. [PubMed: 21866084]

4. Reiter PL, McRee AL, Kadis JA, et al. HPV vaccine and adolescent males. Vaccine. 2011; 29(34): 5595-602. [PubMed: 21704104]

5. Brabin L, Roberts SA, Stretch R, et al. Uptake of first two doses of human papillomavirus vaccine by adolescent schoolgirls in Manchester: prospective cohort study. BMJ. 2008; 336(7652):1056-8. [PubMed: 18436917]

6. Brotherton JM, Deeks SL, Campbell-Lloyd S, et al. Interim estimates of human papillomavirus vaccination coverage in the school-based program in Australia. Commun Dis Intell. 2008; 32(4): 457-61. [PubMed: 19374275]

7. Reeve C, De La Rue S, Pashen D, et al. School-based vaccinations delivered by general practice in rural north Queensland: an evaluation of a new human papilloma virus vaccination program. Commun Dis Intell. 2008; 32(1):94-8. [PubMed: 18522312]

8. Hayes, KA.; Entzel, P.; Berger, W., et al. Working Paper. Early lessons learned from extramural vaccination programs that offer HPV vaccine in US schools. 
9. Schaffer SJ, Fontanesi J, Rickert D, et al. How effectively can health care settings beyond the traditional medical home provide vaccines to adolescents? Pediatrics. 2008; 121 (Suppl 1):S35-45. [PubMed: 18174319]

10. Rothholz, MC. Pharmacists: access to immunization services. Presentation to the 1st National Stakeholder Meeting: review of priorities in the National Vaccine Plan. 2008. Available at: http://www.iom.edu/Activities/PublicHealth/NatVaxPlan/2008-JUL-24.aspx

11. Skiles MP, Cai J, English A, et al. Retail pharmacies and adolescent vaccination--an exploration of current issues. J Adolesc Health. 2011; 48(6):630-2. [PubMed: 21575825]

12. Reiter PL, McRee AL, Gottlieb SL, et al. Correlates of receiving recommended adolescent vaccines among adolescent females in North Carolina. Hum Vaccin. 2011; 7(1):67-73. [PubMed: 21263224]

13. Rand CM, Shone LP, Albertin C, et al. National health care visit patterns of adolescents: implications for delivery of new adolescent vaccines. Arch Pediatr Adolesc Med. 2007; 161(3): 252-9. [PubMed: 17339506]

14. Weiss TW, Zimet GD, Rosenthal SL, et al. Human papillomavirus vaccination of males: attitudes and perceptions of physicians who vaccinate females. J Adolesc Health. 2010; 47(1):3-11. [PubMed: 20547286]

15. Kadis JA, McRee AL, Gottlieb SL, et al. Mothers' support for voluntary provision of HPV vaccine in schools. Vaccine. 2011; 29(14):2542-7. [PubMed: 21300097]

16. Middleman AB, Tung JS. Urban middle school parent perspectives: the vaccines they are willing to have their children receive using school-based immunization programs. J Adolesc Health. 2010; 47(3):249-53. [PubMed: 20708563]

17. Middleman AB, Tung JS. At what sites are parents willing to have their 11 through 14-year-old adolescents immunized? Vaccine. 2010; 28(14):2674-8. [PubMed: 20085835]

18. Rand CM, Humiston SG, Schaffer SJ, et al. Parent and adolescent perspectives about adolescent vaccine delivery: practical considerations for vaccine communication. Vaccine. 2011; 29(44): 7651-8. [PubMed: 21839793]

19. Dempsey AF, Singer D, Clark SJ, Davis MM. Parents' views on 3 shot-related visits: implications for use of adolescent vaccines like human papillomavirus vaccine. Acad Pediatr. 2009; 9(5):34852. [PubMed: 19487172]

20. Dennis, JM. KnowledgePanel design summary. 2011. Available at: http://www.knowledgenetworks.com/knpanel/docs/KnowledgePanel(R)-Design-SummaryDescription.pdf

21. Reiter PL, Cates JR, McRee AL, et al. Statewide HPV vaccine initiation among adolescent females in North Carolina. Sex Transm Dis. 2010; 37(9):549-56. [PubMed: 20414146]

22. Irwin CE Jr, Adams SH, Park MJ, et al. Preventive care for adolescents: few get visits and fewer get services. Pediatrics. 2009; 123(4):e565-72. [PubMed: 19336348]

23. Bilukha OO, Rosenstein N. National Center for Infectious Diseases, Centers for Disease Control and Prevention (CDC). Prevention and control of meningococcal disease. recommendations of the Advisory Committee on Immunization Practices (ACIP). MMWR Recomm Rep. 2005; 54(RR-7): 1-21. [PubMed: 15917737]

24. Broder KR, Cortese MM, Iskander JK, et al. Preventing tetanus, diphtheria, and pertussis among adolescents: use of tetanus toxoid, reduced diphtheria toxoid and acellular pertussis vaccines recommendations of the Advisory Committee on Immunization Practices (ACIP). MMWR Recomm Rep. 2006; 55(RR-3):1-34.

25. Fiore AE, Uyeki TM, Broder K, et al. Prevention and control of influenza with vaccines: recommendations of the Advisory Committee on Immunization Practices (ACIP), 2010. MMWR Recomm Rep. 2010; 59(RR-8):1-62. [PubMed: 20689501]

26. Immunization Action Coalition. State information. State mandates on immunization and vaccinepreventable diseases. 2011. Available at: http://www.immunize.org/laws/

27. Baker, LC.; Bundorf, MK.; Singer, S., et al. Validity of the survey of health and internet and Knowledge Network's panel and sampling. 2003. Available at: http://www.knowledgenetworks.com/ganp/docs/Appendix $\% 20$ Survey $\% 20$ of $\% 20 \mathrm{Health} \% 20$ and $\% 20$ the\%20Internet.pdf 


\section{A: Parents}

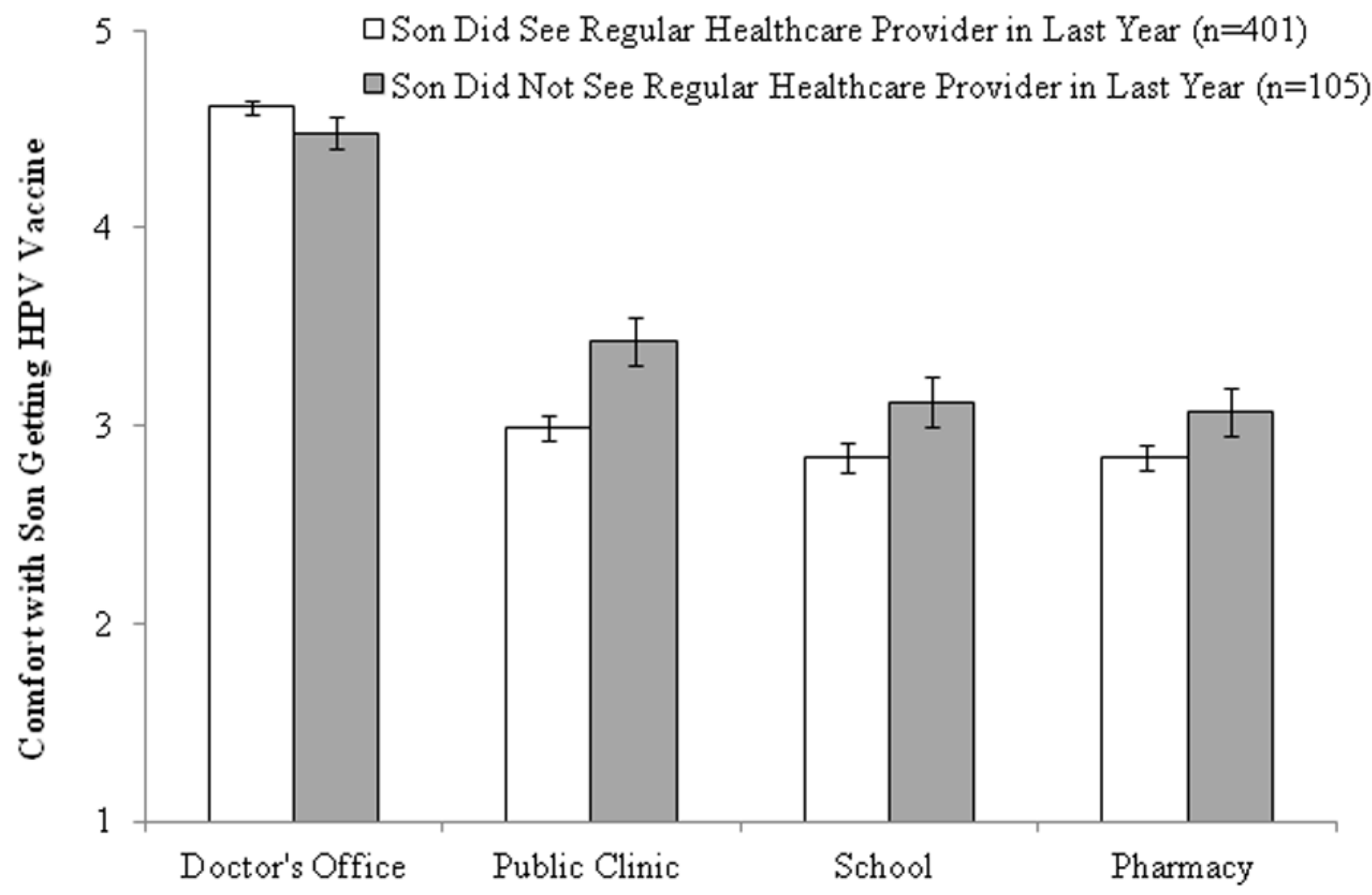




\section{B: Sons}

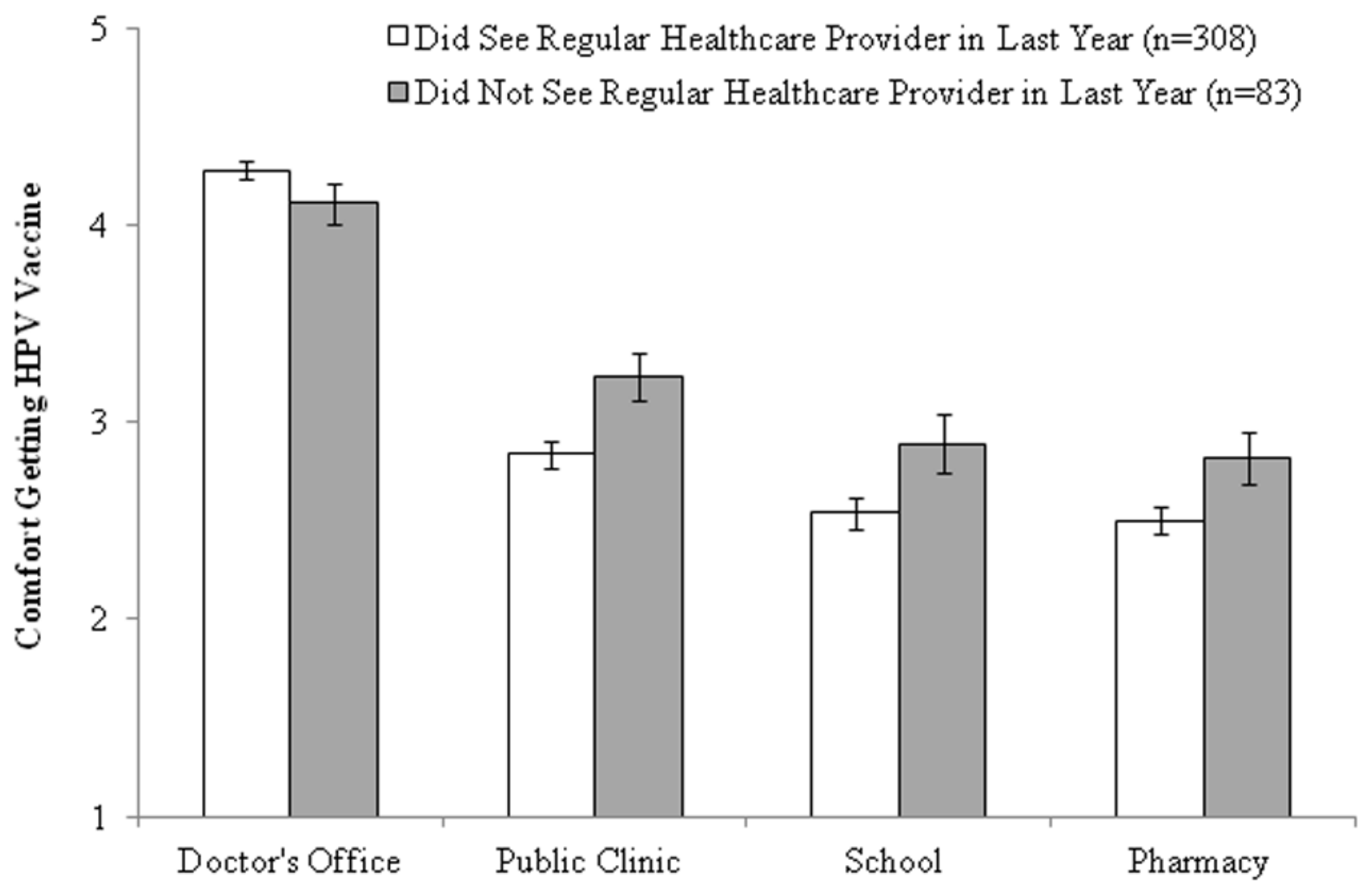

Figure 1.

Comfort with sons getting HPV vaccine in different settings, United States, 2010. Responses by parents (panel A) and sons (panel B). Response scale ranged from "very uncomfortable" (coded as 1) to "very comfortable" (5). Bars indicate the standard errors. 
Table 1

Characteristics of parents $(n=506)$ and their adolescent sons $(n=391)$.

\begin{tabular}{|c|c|}
\hline & $n(\%)$ \\
\hline \multicolumn{2}{|l|}{ Parent Characteristics } \\
\hline \multicolumn{2}{|l|}{ Sex } \\
\hline Female & $271(54)$ \\
\hline Male & $235(46)$ \\
\hline \multicolumn{2}{|l|}{ Age (Years) } \\
\hline$<45$ & $309(61)$ \\
\hline$\geq 45$ & $197(39)$ \\
\hline \multicolumn{2}{|l|}{ Race/Ethnicity } \\
\hline White, Non-Hispanic & $340(67)$ \\
\hline African American, Non-Hispanic & $62(12)$ \\
\hline Hispanic & $78(15)$ \\
\hline Other & $26(5)$ \\
\hline \multicolumn{2}{|l|}{ Education } \\
\hline High School Degree or Less & $223(44)$ \\
\hline Some College or More & $283(56)$ \\
\hline \multicolumn{2}{|l|}{ Household Income } \\
\hline$<\$ 60,000$ & $253(50)$ \\
\hline $2 \$ 60,000$ & $253(50)$ \\
\hline \multicolumn{2}{|l|}{ Son Characteristics ${ }^{a}$} \\
\hline \multicolumn{2}{|l|}{ Age (Years) } \\
\hline $11-12$ & $119(30)$ \\
\hline $13-15$ & $149(38)$ \\
\hline $16-17$ & $123(31)$ \\
\hline \multicolumn{2}{|l|}{ Race/Ethnicity } \\
\hline White, Non-Hispanic & $240(61)$ \\
\hline African American, Non-Hispanic & $48(12)$ \\
\hline Hispanic & $63(16)$ \\
\hline Other & $40(10)$ \\
\hline
\end{tabular}

Son Saw Regular Healthcare Provider in Last Year

\begin{tabular}{lc} 
No & $83(21)$ \\
Yes & $308(79)$ \\
\hline
\end{tabular}

${ }^{a}$ Data collected during parent survey, but we report data only for those sons who completed their own surveys. Sons who completed surveys were similar to non-completers on these characteristics (all $p>0.05$ ). 
